Published in final edited form as:

Lancet Haematol. 2019 August ; 6(8): e419-e428. doi:10.1016/S2352-3026(19)30104-8.

\title{
Ibrutinib plus fludarabine, cyclophosphamide, and rituximab (iFCR) as initial therapy for younger patients with chronic lymphocytic leukaemia: a single-arm, multicentre, phase 2 trial
}

\author{
Matthew S Davids, MD, \\ Dana-Farber Cancer Institute, Department of Medical Oncology, Boston, MA, USA \\ Danielle M Brander, MD, \\ Duke University Medical Center, Department of Medicine, Durham, NC, USA \\ Haesook T Kim, PhD, \\ Dana-Farber Cancer Institute, Department of Data Sciences, Harvard TH Chan School of Public \\ Health, Department of Biostatistics \\ Svitlana Tyekucheva, PhD, \\ Dana-Farber Cancer Institute, Department of Data Sciences, Harvard TH Chan School of Public \\ Health, Department of Biostatistics
}

Jad Bsat,

Dana-Farber Cancer Institute, Department of Medical Oncology, Boston, MA, USA

\footnotetext{
Correspondence to: Matthew S Davids, MD, Department of Medical Oncology, Dana-Farber Cancer Institute, Boston, MA 02215, USA, matthew_davids@dfci.harvard.edu.

MSD, HTK, and JRB were in charge of trial conception and design. MSD, DMB, JMH, JB, KF, AA, LS, MO, CAJ, PA, SN, JC, ASL, JA, EPH, RWT, JSA, DCF, and JRB provided and cared for patients. Collection and assembly of data was conducted by MSD, HTK, SV, JB, and AS, while the data analysis and interpretation was performed by MSD, HTK, SV, and JRB. MSD wrote the first draft of the manuscript and all authors participated in its writing and gave approval for publication.

Declaration of Interests

MSD has received grants from Acerta Pharma, BMS, Genentech, MEI Pharma, Pharmacyclics, Surface Oncology, TG Therapeutics and Verastem, consulting fees from AbbVie, Acerta Pharma, Adaptive Biotechnologies, AstraZeneca, Celgene, Genentech, Gilead Sciences, Janssen, MEI Pharma, Merck, Pharmacyclics, Roche, Syros Pharmaceuticals, TG Therapeutics, and Verastem; DMB has received grants from AbbVie, Pharmacyclics, AstraZeneca, TG Therapeutics, DTRM, BeiGene, MEI Pharma, and Genentech, consulting fees from AbbVie, Teva, AstraZeneca, TG Therapeutics, and Genentech, and non-financial support from AbbVie, Pharmacyclics, Teva, and TG Therapeutics; JB served as an Advisory Board member for Janssen Oncology; KF received research funding from Dana Farber Cancer Institute and consulting fees from Verastem Oncology and AbbVie; AA received consulting fees from AbbVie; CAJ received consulting fees from Kite Pharma, Bayer, Novartis, Pfizer, Celgene, and Humanogen; PA received consulting fees from Merck, BMS, Infinity, Pfizer, Affimed, and Adaptive, and research funding from Merck, BMS, Affimed, Adaptive, Roche, Tensha, Otsuka, and Sigma Tau; ASL received consulting fees from BMS, Humanigen, Research to Practice, and Seattle Genetics; EPH received consulting fees from Intervention Insights; JSA served on the scientific advisory board for Janssen, Kite Pharma, AbbVie, Karyopharm, Bayer, Gilead, Verastem, Merck, Novartis, Juno, Amgen, Celgene, Genentech, and Seattle Genetics; DCF received consulting fees from Merck, Kite, and Gilead; JRB received consulting fees from AbbVie, Acerta, Astellas, AstraZeneca, BeiGene, Celgene, Genentech/Roche, Gilead, Invectys, Janssen, Kite, Loxo, Morphosys, Novartis, Pfizer, Pharmacyclics, Rectx, Sun, Sunesis, Teva, TG Therapeutics, Verastem and Novartis and grants from Gilead, Loxo, Sun and Verastem; and served on the Data Safety Monitoring Boards for Morphosys and Invectys. AS, HTK, JA, JB, JC, JMH, LS, MO, RWT, SN, ST have nothing to disclose.

Data Sharing

Individual participant data that underlie the results reported in this article will be shared after de-identification (text, tables, figures, and appendices). The study protocol will also be made available. These data will become available beginning 9 months following article publication with no end date to investigators whose proposed use of the data has been approved by researchers who provide a methodologically sound proposal to achieve aims in the approved proposal. Proposals should be directed to matthew_davids@dfci.harvard.edu. To gain access, data requestors will need to sign a data access agreement.
} 
Alexandra Savell,

Dana-Farber Cancer Institute, Department of Medical Oncology, Boston, MA, USA

Jeffrey M Hellman, PA-C,

Dana-Farber Cancer Institute, Department of Medical Oncology, Boston, MA, USA

Josie Bazemore, NP,

Dana-Farber Cancer Institute, Department of Medical Oncology, Boston, MA, USA

Karen Francoeur, RN,

Dana-Farber Cancer Institute, Department of Medical Oncology, Boston, MA, USA

Alvaro Alencar, MD,

University of Miami Sylvester Comprehensive Cancer Center, Department of Hematology and Oncology, Miami, FL, USA

Leyla Shune, MD,

University of Kansas Cancer Center, Department of Hematologic Malignancies, Westwood, KS, USA

Mohammad Omaira, MD,

West Michigan Cancer Center, Department of Medical Oncology, Kalamazoo, MI, USA

Caron A Jacobson, MD,

Dana-Farber Cancer Institute, Department of Medical Oncology, Boston, MA, USA

Philippe Armand, MD,

Dana-Farber Cancer Institute, Department of Medical Oncology, Boston, MA, USA

Samuel Ng, MD,

Dana-Farber Cancer Institute, Department of Medical Oncology, Boston, MA, USA

Jennifer Crombie, MD,

Dana-Farber Cancer Institute, Department of Medical Oncology, Boston, MA, USA

Ann S LaCasce,

Dana-Farber Cancer Institute, Department of Medical Oncology, Boston, MA, USA

Jon Arnason, MD,

Beth Israel Deaconess Medical Center, Department of Medical Oncology, Boston, MA, USA

Ephraim P Hochberg, MD,

Massachusetts General Hospital, Department of Medical Oncology, Boston, MA, USA

Ronald W Takvorian, MD,

Massachusetts General Hospital, Department of Medical Oncology, Boston, MA, USA

Jeremy S Abramson, MD,

Massachusetts General Hospital, Department of Medical Oncology, Boston, MA, USA

David C Fisher, MD,

Dana-Farber Cancer Institute, Department of Medical Oncology, Boston, MA, USA

Jennifer R Brown, MD 
Dana-Farber Cancer Institute, Department of Medical Oncology, Boston, MA, USA

\section{Blood Cancer Research Partnership of the Leukemia \& Lymphoma Society}

\section{Summary}

Background-Fludarabine, cyclophosphamide, rituximab (FCR) can provide prolonged diseasefree survival for younger, fit patients with chronic lymphocytic leukaemia (CLL) with mutated $I G H V$; however, patients with unmutated $I G H V$ rarely have durable responses. Ibrutinib is highly effective for patients irrespective of $I G H V$ mutation status but requires continuous therapy. We hypothesised that time-limited ibrutinib plus FCR (iFCR) would induce deep, durable responses in younger, fit patients with CLL.

Methods-We performed a multicentre, open-label, non-randomised, phase 2 trial of iFCR in patients aged $\$ 65$ years with previously untreated CLL without restriction by IGHV mutation status. Ibrutinib was given at $420 \mathrm{mg} / \mathrm{d}$ for 7 days, followed by iFCR for up to six cycles.

Responders continued on ibrutinib maintenance, and patients with undetectable bone marrow minimal residual disease (BM-uMRD) after 2 years were able to discontinue therapy. The primary endpoint was complete response (CR) with BM-uMRD 2 months after FCR. The trial is registered with ClinicalTrials.gov and is still ongoing ().

Findings-Between October 2014 and April 2018, 85 patients with CLL were enrolled in the ITT population at nine US sites. The median age was 55 years (IQR 8). The percentage of patients who achieved CR with BM-uMRD 2 months post-iFCR was 33\% (28/85, 95\% CI 0.23-0.44, $\mathrm{p}=0.0035$ ) compared to the $20 \%$ historical rate with FCR. The best BM-uMRD rate was $84 \%$ (71/85), which did not differ by $I G H V$ mutation status. With a median follow-up of 16.5 months (range 3.1-48.9), one patient progressed and one died. The most common all-grade toxicities were haematologic, including thrombocytopaenia $(74 \%, 63 / 85)$ neutropaenia $(62 \%, 53 / 85)$, and anaemia $(49 \%, 41 / 85)$. Grade $\geq 3$ serious AEs included atrial fibrillation (gr3 3.5\%, 3/85) and Pneumocystis jiroveci pneumonia (gr3, 2.4\%, 2/85).

Interpretation-The proportion of patients who achieved BM-uMRD with iFCR, is, to our knowledge, the highest ever published in patients with CLL unrestricted by prognostic marker status. iFCR is promising as a time-limited novel agent combination regimen for frontline CLL treatment in fit patients.

\section{Introduction}

Chronic lymphocytic leukaemia is the most frequent form of leukaemia diagnosed in developed countries and historically considered an incurable disease. ${ }^{1}$ For fit, younger ( $\$ 65$ years) patients without TP53 aberrations, the chemoimmunotherapy regimen of fludarabine, cyclophosphamide, and rituximab (FCR) has been the gold standard frontline treatment. ${ }^{2,3}$

Recently, long-term follow-up from two prospective clinical trials and one large retrospective study of $\mathrm{FCR}^{4,5,6}$ independently confirmed a plateau in progression-freesurvival (PFS) for patients with the immunoglobulin heavy-chain variable region (IGHV) mutated subtype of chronic lymphocytic leukaemia. Most such patients ( 80\%) who achieved undetectable minimal residual disease (uMRD) remained progression free at 12.8 years, representing a functional cure; however, the proportion of patients treated with FCR 
who achieve complete response (CR) with bone marrow uMRD (BM-uMRD) is only $20 \%$.

${ }^{7}$ Although achieving uMRD in patients with unmutated $I G H V$ can also result in prolonged remissions ( $\sim 40 \%$ at 7 years), this group does not demonstrate a plateau in PFS. The development of regimens with higher rates of sustained remissions for younger chronic lymphocytic leukaemia patients could extend life by decades.

Ibrutinib is an oral, irreversible inhibitor of Bruton tyrosine kinase (BTK), which disrupts Bcell receptor signalling in chronic lymphocytic leukaemia cells, leading to profound antitumour activity. ${ }^{8}$ Like FCR, frontline ibrutinib treatment leads to high response rates; however, unlike FCR, ibrutinib achieves sustained responses independent of $I G H V$ status and other high-risk markers. ${ }^{9-11}$ The randomized phase 3 Eastern Cooperative Oncology Group (ECOG)-1912 study recently found that ibrutinib plus rituximab followed by continuous ibrutinib led to improved PFS and overall survival (OS) compared with FCR in younger chronic lymphocytic leukaemia patients, ${ }^{12}$ establishing ibrutinib as a new standard treatment option in this subpopulation. However, ibrutinib with or without rituximab rarely results in UMRD, and therefore ibrutinib is used as continuous therapy until progression or intolerance. For younger patients, indefinite therapy may be problematic for several reasons, including the risk of toxicities, ${ }^{13}$ the potential for acquisition of resistance mutations, ${ }^{14,15}$ and substantial cost. ${ }^{16}$ As such, novel combination approaches to deepen response and facilitate time-limited therapy are needed. Our group previously piloted a regimen of ibrutinib plus FCR (iFCR) in three younger, fit chronic lymphocytic leukaemia patients, all of whom tolerated the regimen well and achieved deep responses. ${ }^{17}$

Given the already-demonstrated curative potential of FCR in some mutated $I G H V$ patients with chronic lymphocytic leukaemia, along with the ability of ibrutinib to overcome higherrisk prognostic markers, and success in our pilot study, we initiated the phase 2 iFCR trial in younger, fit patients with previously-untreated chronic lymphocytic leukaemia unrestricted by $I G H V$ mutation status.

\section{Methods}

\section{Study design and patients}

This open-label, single-arm, phase 2, multicentre, investigator-initiated study was conducted at nine institutions throughout the United States. Eligible patients were 18 to 65 years at study entry, had a confirmed diagnosis of chronic lymphocytic leukaemia or small lymphocytic lymphoma requiring initial therapy per International Workshop on Chronic Lymphocytic Leukemia (iwCLL) 2008 criteria, ${ }^{18}$ with ECOG PS 0 or 1 . The study was initially designed to accrue 35 patients unrestricted by prognostic marker status-including allowing TP53 aberration. Acceptable baseline laboratory values and key clinical criteria necessitated for study enrolment are defined in the supplemental materials. Given promising data in the initial patients without TP53 aberration, a study amendment added 50 additional patients without del(17p) or TP53 mutation. The protocol was approved by the IRB at each institution, and all patients provided written informed consent. The study was designed according to Good Clinical Practice guidelines and the Declaration of Helsinki. The trial is registered with ClinicalTrials.gov (). 


\section{Procedures}

In the lead-in period, patients were treated with single-agent ibrutinib (420 mg orally, once daily) for 7 days. Starting on cycle 1 , day 1 , fludarabine $\left(25 \mathrm{mg} / \mathrm{m}^{2}\right.$, days $\left.1-3\right)$, cyclophosphamide $\left(250 \mathrm{mg} / \mathrm{m}^{2}\right.$, days $\left.1-3\right)$, and rituximab $\left(375 \mathrm{mg} / \mathrm{m}^{2}\right.$ day 1 , cycle $1 ; 500$ $\mathrm{mg} / \mathrm{m}^{2}$ day 1 in cycles $2-6$ ) were administered with continuous ibrutinib (420 mg orally, once daily) for up to six 28-day cycles. Patients achieving response 2 months after combination continued on ibrutinib $420 \mathrm{mg}$ daily maintenance for up to 2 years (figure 1). After 2 years, patients in the initial cohort with MRD detectable in the bone marrow (BM) continued ibrutinib; those who had uMRD in the BM (BM-uMRD) had the option to discontinue ibrutinib. The 50 patients after the study amendment were required to discontinue maintenance at 2 years if they were BM-uMRD. Patients could resume ibrutinib if they subsequently had MRD detectable in the peripheral blood (PB) or progressed clinically. Patients completing at least three FCR cycles who discontinued early because of FCR-related toxicity were permitted to continue on ibrutinib. Prophylaxis with granulocyte colony-stimulating factor, trimethoprim/sulfamethoxazole or equivalent, and acyclovir or equivalent was mandatory. Pharmacokinetic assays were performed at Frontage Laboratories (Exton, PA, USA) only for patients at the Dana-Farber Cancer Institute and Massachusetts General Hospital, with samples pre-ibrutinib, then on day 1 of iFCR combination therapy predose and 1,2, 4, and 6 hours after ibrutinib.

\section{Outcomes}

The primary endpoint for part 1 of the study was percentage of patients achieving CR with BM-uMRD assessed approximately 2 months after completion of iFCR. The primary endpoint for the second part of the study was MRD-negativity 2 years after ibrutinib discontinuation in patients who achieve BM MRD-negativity after iFCR induction and 2 years of ibrutinib maintenance. Patients with both $\mathrm{CR}$ and $\mathrm{CR}$ with incomplete count recovery (CRi) were considered to have met this endpoint. Secondary endpoints included clinical response rates (overall response rate (ORR), CR, PR), PFS, OS, safety/tolerability, ibrutinib pharmacokinetics, and association of established CLL prognostic factors (local testing) with clinical response (Supplemental Materials). Post-hoc analyses of rates of best clinical response and best BM and PB-MRD undetectability were also assessed. Analysis of additional protocol-specified exploratory outcomes including novel prognostic factors, pharmacodynamics, and a comparison of MRD by flow cytometry with next generation sequencing testing are ongoing and will be reported separately.Response by 2008 iwCLL criteria $^{18}$ and BM-MRD were assessed after three cycles and 2 months after the final FCR cycle, with CT imaging and PB-MRD assessment every 6 months thereafter. BM-MRD was also evaluated 1 year (optional) and 2 years (mandatory) post-iFCR. uMRD was defined as $<10^{-4}$ CLL cell frequency as measured by four-colour flow cytometry in a centralised laboratory. Immunoglobulin levels and CD4+ T-cell counts were not collected for this study.

Adverse events were assessed using Common Terminology Criteria for Adverse Events version 4.03 and 2008 iwCLL criteria for haematologic toxicity. ${ }^{18}$ 


\section{Statistical analysis}

An initial design required 35 patients to estimate the primary endpoint, with a pre-defined efficacy goal of 11 or more of the first 35 patients achieving the primary endpoint. After this initial goal was met (12 responses), the study was amended to allow an additional 50 patients to enrol. The total of 85 patients was based on the expectation that 60 patients would eventually be eligible for post-2-year ibrutinib maintenance evaluation (see Supplemental). The percentage of patients achieving response was compared to historical rates (20\%) using a one-sided exact binomial test, comparisons between $I G H V$ mutated and unmutated groups were performed using Fisher's exact test. Pharmacokinetic data were analyzed using the R package "PKNCA". Safety and response evaluable populations consisted of all patients who received $\geq 1$ dose of study treatment in an ITT analysis.

\section{Role of the funding source}

The study was designed by the investigators and funded by Pharmacyclics LLC (an AbbVie Company), which also provided funding for medical writing assistance. The funder had no role in data collection, analysis, or interpretation. The Leukemia \& Lymphoma Society provided support through the Blood Cancer Research Partnership. The corresponding author had full access to all study data and had final responsibility to submit for publication.

\section{Results}

Between October 23, 2014, and April 23, 2018, 85 patients were enrolled and began study treatment. The median age was 55 years (range 38-65, IQR 8), and 40 of 82 (49\%) patients had Rai stage III or IV disease at study entry (table 1). Additionally, 39 of 82 (48\%) of patients met iwCLL treatment criteria based on bulky, rapidly-progressive, or symptomatic lymphadenopathy (Rai stage 1) or splenomegaly (Rai stage 2). IGHV was unmutated in 46 of $79(58.2 \%)$ patients; del(17p) and TP53 mutations were detected in four of $83(4.8 \%)$ and three of $81(3.7 \%)$ patients, respectively; two of these patients had both. Complex cytogenetics on stimulated karyotype were present in 14 of $83(16.9 \%)$ patients, including all four patients with del(17p). Del(11q) was present in 17 of $83(20.5 \%)$ patients, and NOTCH1 was mutated in five of $58(8.6 \%)$ patients tested. The median baseline $\beta_{2^{-}}$ microglobulin was $3.0 \mathrm{mg} / \mathrm{L}$ (IQR 2.2).

Some 62/85 (73\%), 4 (5\%), 7 (8\%), 11 (13\%), and 1 (1\%) of patients completed six, five, four, three, or one cycle of iFCR therapy, respectively, for a median of six (range 1-6, IQR 1) cycles. Three patients discontinued treatment prior to assessment of the primary endpoint: two because of early toxicity events (grade 3 transaminitis and grade 2 atypical mycobacterial lung infection) and one because of non-compliance. The median number of maintenance ibrutinib cycles at the time of analysis was 11 (range 0-44), at which time maintenance was still ongoing for 52 patients. Three patients withdrew after the combination phase: one (on ibrutinib maintenance) because of sudden death presumed to be cardiac in nature, and two because of patient decision. As of the data cut-off on January 2, 2019, the median follow-up time was 16.5 months (IQR 23.5), with 53 and 34 patients having passed the 1 and 2 year timepoints on ibrutinib maintenance, respectively. 
The trial met its primary endpoint: the estimated combined percentage of patients achieving of $\mathrm{CR}$ or $\mathrm{CRi}$ and $\mathrm{BM}-\mathrm{uMRD}(\mathrm{CR} / \mathrm{CRi} / \mathrm{BM}-\mathrm{uMRD})$ at the post-iFCR response evaluation was $33 \%(28 / 85,95 \%$ CI $0 \cdot 23-0 \cdot 44, p=0.0035$ compared to the $20 \%$ historical rate with FCR), with all patients included in this ITT analysis. CR/CRi/BM-uMRD status was achieved in 37 (44\%) patients at some point, with the number of patients achieving response increasing over the course of combination treatment (figure 2A). CR/CRi/BM-uMRD status was achieved in three of five (60\%) patients with NOTCH1 mutations but not in any of the four patients with del(17p) with complex karyotype or TP53 mutations. Four of ten (40\%) patients with complex karyotype without del(17p) attained this status. CR/CRi/BM-uMRD status was attained in 14 of $33(42.4 \%)$ and 18 of $46(39.1 \%)$ patients with mutated and unmutated $I G H V$, respectively. No statistically significant differences in percentage of patients achieving CR/CRi/BM-uMRD were found in these subgroups at any assessment (supplementary figure 2).

Of the 34 patients who have reached the 2-year post-iFCR ibrutinib maintenance timepoint thus far, 20 (59\%) had BM-uMRD at that time, and 6 (18\%) were MRD-detectable. Eight patients previously had BM-uMRD but did not have repeat bone marrow sampling when they reached the 2-year post-iFCR maintenance timepoint. Of note, there are still 36 patients on ibrutinib maintenance who have not yet reached this timepoint and will be evaluated in the future. In the 19 patients with follow-up PB-MRD test results available beyond the 2year maintenance timepoint, ten continued maintenance (including two patients with uMRD from the initial cohort who elected to continue ibrutinib) and nine discontinued ibrutinib. None of these nine patients who discontinued ibrutinib have yet reached the primary endpoint for the second part of the study (proportion of patients still UMRD 2 years after ibrutinib discontinuation). At a median follow-up of 11.3 months (IQR 6.6) postdiscontinuation for these nine patients, eight (89\%) remained with PB-uMRD, and one patient with pre-treatment del(11q) in $82 \%$ of cells and del(17p) in $7 \%$ of cells with wildtype $T P 53$ and $I G H V$ unmutated (0\%, VH3-43) disease developed detectable PB-MRD and eventual clinical predominantly nodal disease progression 1 year after completing 2 years of ibrutinib maintenance, with plans to resume ibrutinib. No patients have developed Richter syndrome.

Best response of CR, CRi, or PR at any time point was achieved by $84 / 85$ (98.8\%) of the ITT population (table 2). The percentage of patients achieving CR/CRi increased over the course of treatment (figure 2A), with 34\% 2-months post-iFCR increasing to 66\% CR/CRi after additional ibrutinib maintenance therapy. The median time to CR/CRi for the entire study population was 10 months (range 2.8-2.4), with a median of 8 and 12 months in patients with mutated and unmutated $I G H V$, respectively. Responses by genomic subtype are reported in supplementary table 1. CR/CRi at 3 months, 2 months post-iFCR, and at time of best response were $21.2 \%$ (7/33), $42.4 \%$ (14/33), and 78.8\% (26/33), respectively, in patients with mutated $I G H V$ (supplementary table 2; figure 2B) and were $15.2 \%(7 / 46)$, $28.3 \%$ (13/46), and 54.3\% (25/46), respectively, in those with unmutated $I G H V$ (supplementary table 2; figure 2C). Of the 53 patients who have reached 1 year of ibrutinib maintenance so far, 13/37 (35\%) of patients who were in PR post-iFCR converted to CR after 1 year of ibrutinib maintenance. Of the 34 patients who have reached 2 years of ibrutinib maintenance so far, 4/14 (29\%) of patients who were in PR at 1 year post iFCR 
converted to CR after a second year of ibrutinib maintenance. The best CR/CRi on study was significantly higher in the mutated $I G H V$ group (odds ratio 3.08; 95\% CI 1.03-10.15; $\mathrm{p}=0.03$ ), but not after three cycles or at the 2-month post-iFCR response assessment (supplementary figure 2).

BM-uMRD was achieved by 40 (47.1\%), 66 (77.6\%), and $71(83.5 \%)$ of patients after 3 cycles of iFCR, 2-months post-iFCR, and as best response, respectively (table 2 and figure 2). The median time to BM-uMRD was 3 months (range 2.8-28.7) for both patients with mutated and unmutated $I G H V$ (BM-uMRD by genomic subtype in supplementary table 1). In patients with mutated $I G H V$, the percentage of patients achieving BM-uMRD were $48.5 \%$ (16/33), $78.8 \%$ (26/33), and $81.8 \%$ (27/33) at 3 months, post-iFCR, and as best response, respectively (figure $2 \mathrm{~B}$ ), while the corresponding percentages for patients with unmutated $I G H V$ were $41.3 \%$ (19/46), $76 \cdot 1 \%$ (35/46), and 82.6\% (38/46, figure 2C). No demonstrable differences in the percent of patients achieving BM-uMRD were observed between the $I G H V$-mutated and $I G H V$-unmutated groups at any assessment (supplementary figure 2). In a post-hoc analysis, after the exclusion of three patients who were not evaluated for BM-MRD and the four patients with del(17p), BM-uMRD was achieved at least one time during the study in 71 of $78(91 \%)$ patients.

Pharmacokinetic analyses of ibrutinib were performed in 64 patients (supplemental table 3 and supplemental figure 1A). The median area under the curve for iFCR was 337.2 (IQR 198.6-553.9), and the median maximum concentration was $97 \cdot 3$ (IQR 65.1-168.8). Ibrutinib concentration in the first 6 hours after combination dosing with FCR was analysed for association with clinical response and achievement of BM-uMRD (supplemental figure 1AC). Lower AUC (supplemental figure 1D) and Cmax (supplemental figure 1E) were associated with lower likelihood of achieving BM-uMRD 2 months post FCR ( $\mathrm{p}=0.057$ and $\mathrm{p}=0.037$, respectively).

All patients experienced at least one adverse event, of which the majority were grade 1 or 2 (table 3 ). The toxicities were primarily haematologic, most frequently thrombocytopaenia (any grade 74\% (63/85); 32\% (27/85) grade $\geq 3$ ), neutropaenia (any grade 62\% [53/85]; 35\% [30/85] grade 3 ), and anaemia (any grade 49\% [41/85]; $11 \%$ [9/85] grade 3). Eight (9\%) patients experienced grade $>3$ febrile neutropaenia. The most common non-haematologic toxicities of any grade were nausea $(61 / 85,72 \%)$, hyperglycaemia $(54 / 85,64 \%)$, and fatigue (50/85, 59\%); those of grade $\geq 3$ were hyperglycaemia $(6 / 85,7 \%)$, increased aspartate aminotransferase/alanine aminotransferase (each 4/85, 5\%), and lung infection (4/85 5\%). Forty-two (49\%) patients required at least one delay in FCR administration, 19 (22\%) patients required at least one FCR dose reduction, and five (6\%) patients discontinued FCR therapy prior to receiving six cycles because of non-haematologic adverse events. Thirtythree of $85(38.8 \%)$ patients required at least one hold of ibrutinib: 29/85 (34\%) because of toxicity and 4/85 (5\%) because of peri-procedure holding. Eight of $85(9 \%)$ patients required a reduction in ibrutinib dose, and one patient re-escalated back to the full dose. Three of 85 (4\%) patients discontinued ibrutinib during maintenance, two patients in BM-uMRD CR who withdrew and one with persistent arthralgias. Five additional patients discontinued therapy earlier than the planned course because of withdrawal of consent [ $n=3]$ or nonadherence $[\mathrm{n}=2]$ ). In a post-hoc analysis, there was no difference in the proportion who 
achieved best BM-uMRD CR/CRi in patients who had ibrutinib dose interruption or delays due to toxicity compared to those who did not (12/29 (41\%) vs. 25/56 (45\%), OR 1.14 (95\% CI $0 \cdot 42,3 \cdot 15, \mathrm{p}=0.82$ ).

Non-haematologic grade $\geq 3$ serious adverse events (table 4) occurring in more than one patient included atrial fibrillation (grade 3, n=3), and Pneumocystis jiroveci pneumonia (grade 3, n=2; one was taking atovaquone and one was holding prophylaxis at the time of infection). Six patients (7\%) experienced secondary malignancies while on study, all of which were skin cancers (basal cell carcinoma $(n=4)$, squamous cell carcinoma and melanoma in situ ( $\mathrm{n}=1$ each). To date, one patient has died, and this was a sudden death presumed to be cardiac in aetiology, occurring 17 months into ibrutinib maintenance in a patient without significant cardiac risk factors who was in CR-BM-uMRD. Otherwise, the adverse events associated with this regimen were generally effectively managed with dose reductions and treatment interruptions per the standard of care for each of the agents.

\section{Discussion}

With the recent approval of several effective novel agents to treat chronic lymphocytic leukaemia, including inhibitors of BTK, BCL-2, and newer anti-CD20 monoclonal antibodies - a number of innovative approaches to frontline therapy are being evaluated.

For younger, fit patients, the potential for a time-limited combination approach incorporating novel agents is appealing, and developing regimens that achieve deep, durable remissions is now a realistic goal. In this frontline study of younger, fit patients with chronic lymphocytic leukaemia, iFCR induced deep responses; 33\% (28/85) of patients achieved CR/CRi with BM-uMRD 2 months post-iFCR, which appears to be differentiated from the historic $20 \%$ rate with FCR alone. ${ }^{7}$ The 2-year time-limited ibrutinib maintenance portion of the study did appear to be important, as BM-uMRD deepened over that time. To the best of our knowledge, the $84 \%$ (71/85) best BM-uMRD is the highest ever published for any regimen in a CLL population with both high and low risk markers.

Achieving UMRD historically has been associated with prolonged PFS and OS after FCR, and is a better predictor of survival post-iFCR than IW-CLL response status. ${ }^{20}$ Thus, despite the fact that our percent of patients achieving CR with BM-uMRD is only modestly higher than FCR alone, the substantially higher proportion of patients who achieved BM-uMRD suggests that PFS after iFCR may be longer than FCR alone, although additional studies are needed for confirmation. Although the median follow-up of 16.5 months is still relatively short, only one progression event has occurred to date in the 85 patients treated. This patient (with unmutated $I G H V$ ) is one of nine who has thus far discontinued ibrutinib after achieving BM-uMRD after 2 years of ibrutinib maintenance. After a median follow-up of 11.3 months since ibrutinib discontinuation, none of the other eight 8 patients reaching BMuMRD has developed detectable PB-MRD. This suggests the potential for a time-limited ibrutinib-based combination regimen for frontline therapy in younger chronic lymphocytic leukaemia patients. Deep remission and discontinuation of therapy provide reductions in toxicity, follow-up visits, likelihood of disease resistance, and cost. As such, iFCR is a promising new regimen with the advantage of being potentially time-limited, in contrast to 
ibrutinib plus rituximab, which is also highly effective but requires continuous therapy for this young, fit population. ${ }^{12}$

iFCR demonstrated a favourable safety profile, with relatively few grade $\geq 3$ adverse events and few discontinuations. The percentage $(62 / 85,73 \%)$ of patients completing all 6 cycles of iFCR is very similar to the $74 \%$ observed with FCR alone in the CLL8 study ${ }^{2}$, suggesting that ibrutinib does not impact the ability to deliver a full course of FCR therapy. The incidence of grade $\geq 3$ haematologic toxicities were comparable overall to those observed historically with FCR alone, although our incidence of severe febrile neutropenia $(8 / 85,9 \%)$ is lower than that seen historically with FCR (generally $>20 \%$ ). This reduction in infectious complications may be due primarily to the mandatory use of granulocyte colony-stimulating factor support in our study, along with both antiviral and Pneumocystis jiroveci pneumonia prophylaxis. The incidence of several commonly-reported ibrutinib-related adverse events were low and similar to those seen in the E1912 study, ${ }^{12}$ including grade 3 or higher infection, atrial fibrillation, and major bleeding in $9(10.6 \%), 3(3.5 \%)$, and $0 \%$ of patients, respectively; one sudden death occurred. Most patients were able to receive all six cycles of FCR, and few have had to discontinue maintenance prior to the end of the 2-year course. The pharmacokinetics of ibrutinib were similar to that seen with ibrutinib monotherapy in prior studies, ${ }^{19}$ suggesting that ibrutinib pharmacokinetics are not altered by FCR.

Another notable aspect of our study is that patients treated with iFCR achieved s BM-uMRD at equivalent percentages irrespective of $I G H V$ mutational status. For patients with mutated $I G H V$, iFCR has the potential to increase the chance of long-term remission. For patients with unmutated $I G H V$, who continuously relapse post-iFCR, the distinct mechanisms of ibrutinib and FCR may result in the potential for a plateau on the PFS curve (as has previously only been seen in mutated $I G H V$ patients). Longer follow-up will be required to evaluate this possibility. We did treat four patients with TP53 aberration in the initial part of the study; although all achieved response, most were partial, with detectable MRD. We therefore excluded these patients from the expansion cohort and we believe that novel-agentonly combination approaches may be better suited for this subpopulation.

Several promising chemotherapy-free, time-limited approaches to frontline chronic leukocytic leukaemia treatment are in development. These include venetoclax plus obinutuzumab, ${ }^{21,22}$ ibrutinib plus venetoclax, ${ }^{23}$ and ibrutinib plus venetoclax plus obinutuzumab. ${ }^{24}$ Each of these regimens has been shown to lead to high levels of patients achieving BM-uMRD, suggesting that these impressive responses may also be durable. However, unlike the very long-term follow-up available from prior studies of FCR alone, follow-up on these studies of chemotherapy free regimens is short, and it is unknown whether these deep initial responses will have the same potential for a plateau in the survival curve that has been demonstrated with FCR. Because iFCR was generally well tolerated in the young, fit population in the current study, it is an appealing time-limited frontline treatment option for such patients, given its potential to result in long-term remission after treatment discontinuation.

There are several limitations to our study. An important consideration for patients choosing FCR-based therapy is the uncommon but serious longer-term risk of secondary myeloid 
neoplasia. None of our 85 patients have experienced this complication at the time of data cut-off. The longest duration of follow-up is 4 years post-iFCR; however, longer follow-up is needed to address this question. Retrospective studies have suggested that an abbreviated course of three cycles of FCR may preserve the efficacy of this regimen if uMRD is achieved, while potentially decreasing toxicity. ${ }^{25}$ Our data demonstrate deepening response in many patients during cycles 4 to 6 of FCR, but our study design cannot address the impact of three vs. six cycles of iFCR. Future studies exploring discontinuation after three cycles of chemoimmunotherapy would be informative, and a promising study of three cycles of FC plus obinutuzumab in combination with ibrutinib is ongoing. ${ }^{26}$

In conclusion, the results of this phase 2 study in frontline chronic lymphocytic leukaemia demonstrated that 6 months of ibrutinib with FCR followed by 2 years of ibrutinib maintenance (iFCR) leads, to the best of our knowledge, to the highest level of patients achieving of BM-uMRD ever published for any regimen used to treat this disease. Moreover, in this younger, fit population, iFCR was generally well tolerated. This time-limited treatment approach may increase the potential of FCR to functionally cure patients with mutated $I G H V$, and, importantly, may for the first time extend this potential to patients with unmutated $I G H V$. Although a randomised phase 3 trial comparing iFCR with chemotherapy-free approaches would be needed to definitively determine the optimal initial regimen for this population, our results represent an initial step toward establishing a new standard of care with curative potential for a broad population of younger, fit patients with chronic lymphocytic leukaemia.

\section{Supplementary Material}

Refer to Web version on PubMed Central for supplementary material.

\section{Acknowledgements}

Dr. Davids acknowledges the Leukemia \& Lymphoma Society for his Scholar in Clinical Research Award. Dr. Brown acknowledges support from the 1U10CA180861-03 (PI Clara Bloomfield). All authors wish to thank Nexus GG Science LLC for medical writing support, in particular Tom Renau, PhD, Devon Roll, PhD, Robert Rydzewski, MS, CMPP, and Emily Hale.

Funding Pharmacyclics, Leukemia \& Lymphoma Society

\section{References}

1. Hallek M, Shanafelt TD, Eichhorst B. Chronic lymphocytic leukaemia. Lancet (London, England) 2018; 391(10129): 1524-37.

2. Hallek M, Fischer K, Fingerle-Rowson G, et al. Addition of rituximab to fludarabine and cyclophosphamide in patients with chronic lymphocytic leukaemia: a randomised, open-label, phase 3 trial. Lancet (London, England) 2010; 376(9747): 1164-74.

3. Eichhorst B, Fink AM, Bahlo J, et al. First-line chemoimmunotherapy with bendamustine and rituximab versus fludarabine, cyclophosphamide, and rituximab in patients with advanced chronic lymphocytic leukaemia (CLL10): an international, open-label, randomised, phase 3, non-inferiority trial. The Lancet Oncology 2016; 17(7): 928-42. [PubMed: 27216274]

4. Thompson PA, Wierda WG. Eliminating minimal residual disease as a therapeutic end point: working toward cure for patients with CLL. Blood 2016; 127(3): 279-86. [PubMed: 26576865] 
5. Fischer K, Bahlo J, Fink AM, et al. Long-term remissions after FCR chemoimmunotherapy in previously untreated patients with CLL: updated results of the CLL8 trial. Blood 2016; 127(2): 208-15. [PubMed: 26486789]

6. Rossi D, Terzi-di-Bergamo L, De Paoli L, et al. Molecular prediction of durable remission after first-line fludarabine-cyclophosphamide-rituximab in chronic lymphocytic leukemia. Blood 2015; 126(16): 1921-4. [PubMed: 26276669]

7. Bottcher S, Ritgen M, Fischer K, et al. Minimal residual disease quantification is an independent predictor of progression-free and overall survival in chronic lymphocytic leukemia: a multivariate analysis from the randomized GCLLSG CLL8 trial. Journal of clinical oncology : official journal of the American Society of Clinical Oncology 2012; 30(9): 980-8. [PubMed: 22331940]

8. Byrd JC, Furman RR, Coutre SE, et al. Targeting BTK with ibrutinib in relapsed chronic lymphocytic leukemia. N Engl J Med 2013; 369(1): 32-42. [PubMed: 23782158]

9. Burger JA, Tedeschi A, Barr PM, et al. Ibrutinib as Initial Therapy for Patients with Chronic Lymphocytic Leukemia. N Engl J Med 2015; 373(25): 2425-37. [PubMed: 26639149]

10. O'Brien S, Furman RR, Coutre S, et al. Single-Agent Ibrutinib in Treatment-Naive and Relapsed/ Refractory Chronic Lymphocytic Leukemia: A 5-Year Experience. Blood 2018; 131(17)19101919 [PubMed: 29437592]

11. Ahn IE, Farooqui MZH, Tian X, et al. Depth and durability of response to ibrutinib in CLL: 5-year follow-up of a phase 2 study. Blood 2018; 131(21): 2357-66. [PubMed: 29483101]

12. Shanafelt TD, Wang V, Kay NE, et al. A Randomized Phase III Study of Ibrutinib (PCI-32765)Based Therapy Vs. Standard Fludarabine, Cyclophosphamide, and Rituximab (FCR) Chemoimmunotherapy in Untreated Younger Patients with Chronic Lymphocytic Leukemia (CLL): A Trial of the ECOG-ACRIN Cancer Research Group (E1912). Blood 2018; 132(Suppl 1): LBA-4.

13. Brown JR. How I treat CLL patients with ibrutinib. Blood 2018; 131(4): 379-86. [PubMed: 29255067]

14. Woyach JA, Furman RR, Liu TM, et al. Resistance mechanisms for the Bruton's tyrosine kinase inhibitor ibrutinib. N Engl J Med 2014; 370(24): 2286-94. [PubMed: 24869598]

15. Woyach JA, Ruppert AS, Guinn D, et al. BTK(C481S)-Mediated Resistance to Ibrutinib in Chronic Lymphocytic Leukemia. Journal of clinical oncology : official journal of the American Society of Clinical Oncology 2017; 35(13): 1437-43. [PubMed: 28418267]

16. Shanafelt TD, Borah BJ, Finnes HD, et al. Impact of ibrutinib and idelalisib on the pharmaceutical cost of treating chronic lymphocytic leukemia at the individual and societal levels. Journal of oncology practice 2015; 11(3): 252-8. [PubMed: 25804983]

17. Brown JR, Barrientos JC, Barr PM et al. The Bruton tyrosine kinase inhibitor ibrutinib with chemoimmunotherpay in ptatients with chronic lymphocytic leukemia. Blood 2015; 125(19):2915-22. [PubMed: 25755291]

18. Hallek M, Cheson BD, Catovsky D, et al. Guidelines for the diagnosis and treatment of chronic lymphocytic leukemia: a report from the International Workshop on Chronic Lymphocytic Leukemia updating the National Cancer Institute-Working Group 1996 guidelines. Blood 2008; 111(12): 5446-56. [PubMed: 18216293]

19. Advani RH, Buggy JJ, Sharman JP, et al. Bruton tyrosine kinase inhibitor ibrutinib (PCI-32765) has significant activity in patients with relapsed/refractory B-cell malignancies. Journal of clinical oncology : official journal of the American Society of Clinical Oncology 2013; 31(1): 88-94. [PubMed: 23045577]

20. Kovacs G, Robrecht S, Fink AM, et al. Minimal Residual Disease Assessment Improves Prediction of Outcome in Patients With Chronic Lymphocytic Leukemia (CLL) Who Achieve Partial Response: Comprehensive Analysis of Two Phase III Studies of the German CLL Study Group. Journal of clinical oncology : official journal of the American Society of Clinical Oncology 2016; 34(31): 3758-65. [PubMed: 27573660]

21. Flinn IW, Gribben JG, Dyer MJS, et al. Safety, Efficacy and MRD Negativity of a Combination of Venetoclax and Obinutuzumab in Patients with Previously Untreated Chronic Lymphocytic Leukemia - Results from a Phase 1b Study (GP28331). Blood 2017; 130(Suppl 1): 430. 
22. Fischer K, Al-Sawaf O, Fink AM, et al. Venetoclax and obinutuzumab in chronic lymphocytic leukemia. Blood 2017; 129(19): 2702-5. [PubMed: 28325865]

23. Jain N, Keating MJ, Thompson PA, et al. Combined Ibrutinib and Venetoclax in Patients with Treatment-Naïve High-Risk Chronic Lymphocytic Leukemia (CLL). Blood 2018; 132(Suppl 1): 696.

24. Rogers KA, Huang Y, Ruppert AS, et al. Phase 2 Study of Combination Obinutuzumab, Ibrutinib, and Venetoclax in Treatment-Naive and Relapsed/Refractory Chronic Lymphocytic Leukemia. Blood 2018; 132(Suppl 1): 693.

25. Strati P, Keating MJ, O'Brien SM, et al. Eradication of bone marrow minimal residual disease may prompt early treatment discontinuation in CLL. Blood 2014; 123(24): 3727-32. [PubMed: 24705492]

26. Jain N, Thompson PA, Burger JA, et al. Ibrutinib, Fludarabine, Cyclophosphamide, and Obinutuzumab (iFCG) for Firstline Treatment of Patients with CLL with Mutated IGHV and without TP53 Aberrations. Blood 2018; 132(Suppl 1): 695. 


\section{Research in context}

\section{Evidence before this study}

The combination of fludarabine, cyclophosphamide, and rituximab (FCR) has curative potential for younger, fit patients with chronic lymphocytic leukaemia who have lowerrisk immunoglobulin heavy-chain variable region $(I G H V)$-mutated disease, and has been a standard of care for frontline therapy in younger patients; however, patients with the higher-risk unmutated $I G H V$ rarely derive long-term benefit. Bruton tyrosine kinase inhibition with ibrutinib was recently shown to be highly effective in younger chronic lymphocytic leukaemia patients irrespective of $I G H V$ mutation status; however, it requires continuous therapy, which is associated with the risk of ongoing toxicities, the potential development of resistance mutations, and substantial cost. To better understand the prior data on ibrutinib and FCR treatment in this patient population, we searched PubMed for all clinical trial publications using the search terms "chronic lymphocytic leukaemia" AND "ibrutinib" AND ("frontline" OR "untreated") AND ("fludarabine cyclophosphamide rituximab" AND "trial."). We found no studies that had previously evaluated this approach. We also searched the online database of abstracts presented at the 2018 Annual Meeting of the American Society of Hematology and found analyses of two ongoing studies evaluating the combination of ibrutinib with chemoimmunotherapy for frontline CLL treatment (ICLL-FILO-07 trial, abstract 1858 and iFCG trial, abstract 695).

\section{Added value of this study}

To our knowledge, this is the first study to have evaluated the efficacy and safety of ibrutinib given in combination with FCR (iFCR) in previously untreated younger, fit patients with chronic lymphocytic leukaemia. Using the International Workshop for Chronic Lymphocytic Leukaemia Criteria, investigator-assessed overall response was achieved in all but one of the 85 patients: $66 \%$ of patients achieved a complete response or complete response with incomplete count recovery (CR/CRi), and $84 \%$ achieved undetectable minimal residual disease in the bone marrow (BM-uMRD) as their best response at any time on trial. With a median follow-up of 16.5 months, responses were durable; only one patient experienced disease progression. The best percentage of patients achieving CR/CRi with BM-uMRD of $44 \%$ is favourable in the context of the $20 \%$ previously reported for FCR alone. To our knowledge, the 84\% BM-uMRD is the highest ever published for a regimen used to treat a broad chronic lymphocytic leukaemia population that includes all prognostic subgroups, including high-risk patients. The safety profile of iFCR was generally favourable; typical toxicities associated with ibrutinib and FCR were observed, but no additional toxicities were discernible with the combination.

\section{Implications of all the available evidence}

The results of our study suggest that iFCR is a promising novel agent-containing, timelimited combination for frontline therapy in fit chronic lymphocytic leukaemia patients. These data represent a first step toward establishing a new standard of care with curative 
potential for younger, fit patients with chronic lymphocytic leukaemia irrespective of $I G H V$ mutation status. 


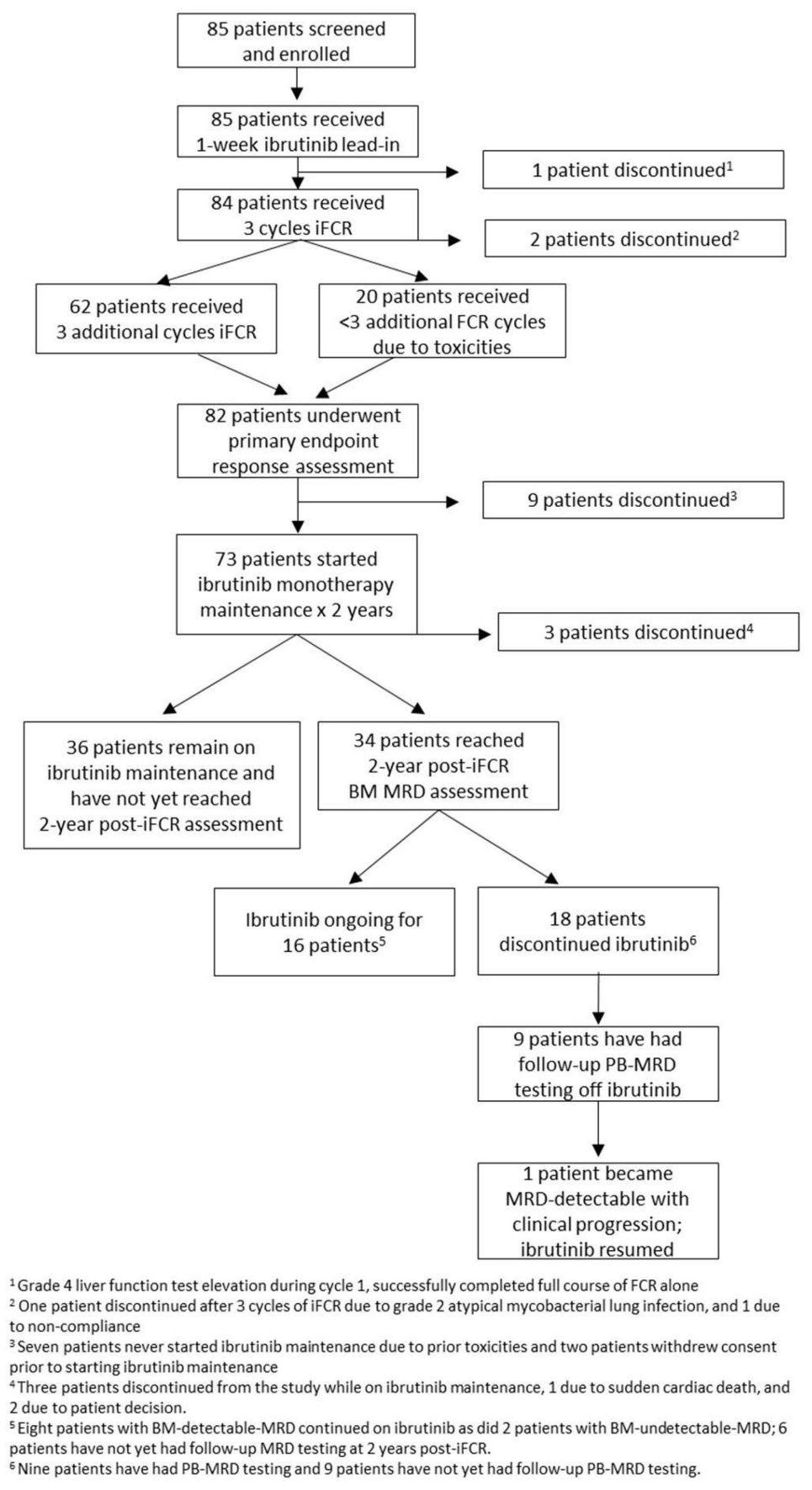

Figure 1: Study schema

$\mathrm{BM}=$ bone marrow. $\mathrm{CR}=$ complete response. $\mathrm{Cri}=$ complete response with incomplete count recovery. $\mathrm{iFCR}=$ ibrutinib in combination with fludarabine, cyclophosphamide, and rituximab. $\mathrm{MRD}=$ minimal residual disease. $\mathrm{PD}=$ progressive disease. $\mathrm{PR}=$ partial response. $\mathrm{Pt}=$ patient. $\mathrm{SD}=$ stable disease. 
A

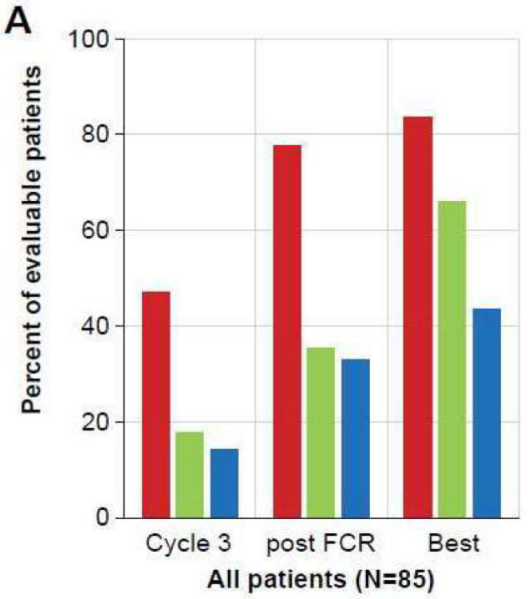

B

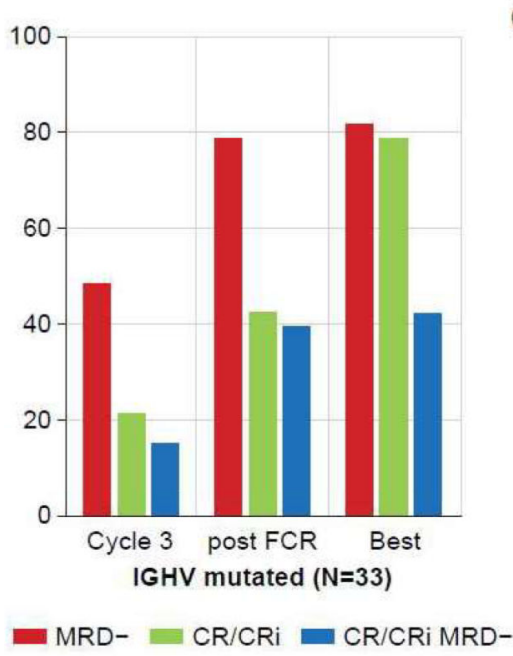

C

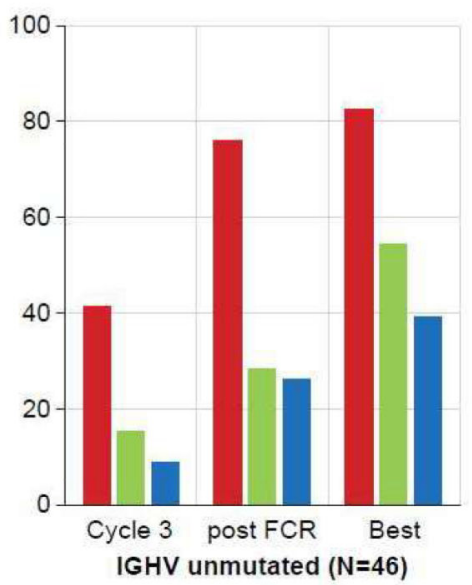

Figure 2: Primary Endpoint: Complete response (CR) or complete response with incomplete count recovery (Cri) and undetectable minimal residual disease (BM-uMRD) by assessement (A) All patients. (B) Patients with mutated immunoglobulin heavy-chain variable region (IGHV). (C) Patients with unmutated IGHV. FCR=fludarabine, cyclophosphamide, plus rituximab. 
Table 1:

Baseline characteristics

\begin{tabular}{|c|c|c|c|}
\hline & Original & Expansion & Total \\
\hline & $\mathrm{N}=35$ & $\mathbf{N}=\mathbf{5 0}$ & $\mathrm{N}=85$ \\
\hline Median age (IQR), years & $55(9.5)$ & $54(7)$ & $55(8)$ \\
\hline Men, n (\%) & $25(71)$ & $31(62)$ & $56(65.9)$ \\
\hline Women & $10(29)$ & $19(38)$ & $29(34 \cdot 1)$ \\
\hline \multicolumn{4}{|l|}{ ECOG performance status, $n(\%)$} \\
\hline $\mathbf{0}$ & $23(66)$ & $27(54)$ & $50(58 \cdot 8)$ \\
\hline 1 & $12(34)$ & $23(46)$ & $35(41 \cdot 2)$ \\
\hline \multicolumn{4}{|l|}{ Rai stage, n (\%) } \\
\hline $\mathbf{0}$ & $1(2 \cdot 9)$ & $0(0)$ & $1(1 \cdot 2)$ \\
\hline 1 & $6(17 \cdot 1)$ & $14(28)$ & $20(23 \cdot 5)$ \\
\hline 2 & $8(22 \cdot 9)$ & $13(26)$ & $21(24 \cdot 7)$ \\
\hline 3 & $4(11 \cdot 4)$ & $7(14)$ & $11(12 \cdot 9)$ \\
\hline 4 & $16(45 \cdot 7)$ & $13(26)$ & $29(34 \cdot 1)$ \\
\hline Median WBC (IQR), × 109/L & $78(146 \cdot 7)$ & $115(136 \cdot 7)$ & $109(146 \cdot 5)$ \\
\hline Median haemoglobin (IQR), g/dL & $12(3 \cdot 2)$ & $11(3 \cdot 3)$ & $12(3 \cdot 3)$ \\
\hline Median haematocrit (IQR), \% & $37(9 \cdot 6)$ & $35(9 \cdot 2)$ & $37(9 \cdot 7)$ \\
\hline Median platelets (IQR), $\times 10^{9} / \mathrm{L}$ & $141(92)$ & $127(74)$ & $127(79)$ \\
\hline Median BM involvement (IQR), \% & $47(37 \cdot 5)$ & $77(31 \cdot 3)$ & $60(40)$ \\
\hline Median IgG (IQR), mg/dL & $753(481)$ & $631(425)$ & $676(463 \cdot 3)$ \\
\hline Median IgA (IQR), mg/dL & $74(52 \cdot 8)$ & $79(47)$ & $77(53 \cdot 5)$ \\
\hline Median $\beta_{2}$-microglobulin (IQR), mg/L & $4(1 \cdot 9)$ & $2(1 \cdot 9)$ & $3 \cdot 0(2 \cdot 2)$ \\
\hline$I G H V$ unmutated & $21 / 32(65 \cdot 6)$ & $25 / 47(53 \cdot 2)$ & $46 / 79(58.2 \%)$ \\
\hline ZAP-70 positive & $21 / 33(63 \cdot 6)$ & $22 / 43(51 \cdot 1)$ & $43 / 76(56.6 \%)$ \\
\hline $\operatorname{del}(17 p)$ & $4 / 33(12 \cdot 1)$ & $0 / 50(0)$ & $4 / 83(4.8 \%)$ \\
\hline $\operatorname{del}(11 q)$ & $9 / 33(27 \cdot 3)$ & $8 / 50(16)$ & $17 / 83(20.5 \%)$ \\
\hline $\operatorname{del}(13 q)$ & $17 / 33(51 \cdot 5)$ & $28 / 50(56)$ & $45 / 83(54 \cdot 2 \%)$ \\
\hline Normal FISH & $5 / 33(15 \cdot 2)$ & $9 / 50(18)$ & $14 / 82(17 \cdot 1 \%)$ \\
\hline Trisomy 12 & $6 / 33(18 \cdot 2)$ & $8 / 50(16)$ & $14 / 83(16.9 \%)$ \\
\hline 6q detected & $1 / 33(3 \cdot 0)$ & $1 / 50(2)$ & $2 / 82(2 \cdot 4 \%)$ \\
\hline Complex cytogenetics & & & $14 / 83(16 \cdot 9 \%)$ \\
\hline Including del(17p) & $3 / 33(9 \cdot 1)$ & $1 / 50(2)$ & $4 / 83(4 \cdot 8 \%)$ \\
\hline Without del(17p) & $4 / 33(12 \cdot 1)$ & $6 / 50(12)$ & $10 / 83(12 \cdot 1 \%)$ \\
\hline TP53 mutation & $2 / 33(6 \cdot 1)$ & $1 / 48(2 \cdot 1)$ & $3 / 81(3 \cdot 7 \%)$ \\
\hline NOTCH1 mutation & $2 / 27(7 \cdot 4)$ & $3 / 31(9 \cdot 7)$ & $5 / 58(8.6 \%)$ \\
\hline
\end{tabular}

BM=bone marrow. ECOG=Eastern Cooperative Oncology Group. FISH=fluorescence in situ hybridisation, non-hierarchical. Ig=immunoglobulin. $I G H V=$ immunoglobulin heavy-chain variable region. $\mathrm{WBC}=$ white blood cell. 
Table 2:

Response, bone marrow MRD, and combined response/bone marrow MRD by assessment

\begin{tabular}{|c|c|c|c|}
\hline & Cycle $3(n=85)$ & Post-iFCR ${ }^{*}(\mathrm{n}=85)$ & Best Response $(\mathrm{n}=85)$ \\
\hline $\mathrm{CR}^{\dagger}$ & $9(10 \cdot 6 \%)$ & $20(23 \cdot 5 \%)$ & $51(60 \cdot 0 \%)$ \\
\hline $\mathrm{CRi}^{\dagger}$ & $6(7 \cdot 1 \%)$ & $10(11.8 \%)$ & $5(5.9 \%)$ \\
\hline PR & $59(69 \cdot 4 \%)$ & $52(61.2 \%)$ & $28(32.9 \%)$ \\
\hline CR or CRi or PR & $74(87 \cdot 1 \%)$ & $82(96 \cdot 5 \%)$ & $84(98 \cdot 8 \%)$ \\
\hline BM-MRD- & $40(47 \cdot 1 \%)$ & $66(77 \cdot 6 \%)$ & $71(83 \cdot 5 \%)$ \\
\hline BM-MRD+ & $38(44 \cdot 7 \%)$ & $14(16 \cdot 5 \%)$ & $14(16 \cdot 5 \%)$ \\
\hline BM-MRD UNK & $7(8 \cdot 2 \%)$ & $5(5.9 \%)$ & 0 \\
\hline CR/MRD- & $5(5.9 \%)$ & $19(22 \cdot 4 \%)$ & $\mathrm{NA}^{t^{t}}$ \\
\hline $\mathrm{CR} / \mathrm{MRD}+$ & $4(4 \cdot 7 \%)$ & $1(1 \cdot 2 \%)$ & $\mathrm{NA}^{t^{t}}$ \\
\hline CRi/MRD- & $4(4 \cdot 7 \%)$ & $9(10 \cdot 6 \%)$ & $\mathrm{NA}^{*}$ \\
\hline CRi/MRD+ & $1(1.2 \%)$ & $1(1 \cdot 2 \%)$ & $\mathrm{NA}^{* t}$ \\
\hline PR/MRD- & $31(36 \cdot 5 \%)$ & $38(44.7 \%)$ & $\mathrm{NA}^{*}$ \\
\hline PR/MRD+ & $28(32.9 \%)$ & $12(14 \cdot 1 \%)$ & $\mathrm{NA}^{* t}$ \\
\hline
\end{tabular}

$\mathrm{BM}-\mathrm{uMRD}=$ minimal residual disease in the bone marrow. $\mathrm{CR}=$ complete response. $\mathrm{CRi}=$ complete response with incomplete count recovery.

$\mathrm{FCR}=$ fludarabine, cyclophosphamide, plus rituximab. $\mathrm{MRD}=$ minimum residual disease. $\mathrm{NA}=$ not applicable. $\mathrm{PR}=$ partial response. UNK=unknown.

Post-iFCR evaluations occurred at 1 month instead of 2 months in seven patients due to scheduling issues.

${ }^{\dagger} \mathrm{CR}$ and CRi were combined when evaluating the primary trial endpoint of BM-uMRD CR post-iFCR.

Because of optional sampling times and missed samples, the best response cannot be defined and are listed as NA. 
Table 3:

All-grade treatment-emergent adverse events in $\geq 10 \%$ of patients and grade $\geq 3$ events in all patients

\begin{tabular}{|c|c|c|c|c|}
\hline $\mathrm{N}=85$ & Grade 1-2, n (\%) & Grade 3, n (\%) & Grade 4, n (\%) & Grade 5, n (\%) \\
\hline \multicolumn{5}{|l|}{ Haematologic } \\
\hline Decreased platelet count & $36(42 \%)$ & $23(27 \%)$ & $4(5 \%)$ & 0 \\
\hline Decreased lymphocyte count & $2(2 \%)$ & $11(13 \%)$ & $40(47 \%)$ & 0 \\
\hline Decreased neutrophil count & $23(27 \%)$ & $12(14 \%)$ & $18(21 \%)$ & 0 \\
\hline Decreased WBC count & $28(33 \%)$ & $18(21 \%)$ & $4(5 \%)$ & 0 \\
\hline Anaemia & $32(38 \%)$ & $9(11 \%)$ & 0 & 0 \\
\hline Increased lymphocyte count & $4(5 \%)$ & $16(19 \%)$ & 0 & 0 \\
\hline Leukocytosis & $1(1 \%)$ & $17(20 \%)$ & 0 & 0 \\
\hline Febrile neutropaenia & 0 & $7(8 \%)$ & $1(1 \%)$ & 0 \\
\hline \multicolumn{5}{|l|}{ Non-haematologic } \\
\hline Nausea & $60(71 \%)$ & $1(1 \%)$ & 0 & 0 \\
\hline Hyperglycaemia & $48(56 \%)$ & $6(7 \%)$ & 0 & 0 \\
\hline Fatigue & $50(59 \%)$ & 0 & 0 & 0 \\
\hline Bruising & $44(52 \%)$ & 0 & 0 & 0 \\
\hline Diarrhoea & $30(35 \%)$ & $2(2 \%)$ & 0 & 0 \\
\hline Constipation & $29(31 \%)$ & 0 & 0 & 0 \\
\hline Arthralgia & $27(32 \%)$ & 0 & 0 & 0 \\
\hline Cough & $27(32 \%)$ & 0 & 0 & 0 \\
\hline Hypocalcaemia & $27(32 \%)$ & 0 & 0 & 0 \\
\hline Acneiform rash & $26(31 \%)$ & 0 & 0 & 0 \\
\hline Increased AST & $21(25 \%)$ & $2(2 \%)$ & $2(2 \%)$ & 0 \\
\hline Hyponatremia & $22(26 \%)$ & $1(1 \%)$ & 0 & 0 \\
\hline Fain in extremity ${ }^{*}$ & $23(27 \%)$ & 0 & 0 & 0 \\
\hline Headache & $21(25 \%)$ & 0 & 0 & 0 \\
\hline Other skin/subcutaneous tissue disorder & $20(24 \%)$ & $1(1 \%)$ & 0 & 0 \\
\hline Myalgia & $20(24 \%)$ & 0 & 0 & 0 \\
\hline Increased ALT & $14(16 \%)$ & $2(2 \%)$ & $2(2 \%)$ & 0 \\
\hline Fever & $18(21 \%)$ & 0 & 0 & 0 \\
\hline Upper respiratory infection & $17(20 \%)$ & $1(1 \%)$ & 0 & 0 \\
\hline Vomiting & $17(20 \%)$ & $1(1 \%)$ & 0 & 0 \\
\hline Hyperkalaemia & $15(18 \%)$ & $2(2 \%)$ & 0 & 0 \\
\hline Insomnia & $17(20 \%)$ & 0 & 0 & 0 \\
\hline Increased blood bilirubin & $14(17 \%)$ & $2(2 \%)$ & 0 & 0 \\
\hline Gastroesophageal reflux disease & $15(18 \%)$ & 0 & 0 & 0 \\
\hline Hypokalaemia & $14(17 \%)$ & $1(1 \%)$ & 0 & 0 \\
\hline Nasal congestion & $15(18 \%)$ & 0 & 0 & 0 \\
\hline Hypophosphatemia & $11(13 \%)$ & $3(4 \%)$ & 0 & 0 \\
\hline Back pain & $13(15 \%)$ & 0 & 0 & 0 \\
\hline
\end{tabular}




\begin{tabular}{|c|c|c|c|c|}
\hline $\mathrm{N}=85$ & Grade 1-2, n (\%) & Grade 3, n (\%) & Grade 4, n (\%) & Grade 5, n (\%) \\
\hline Dry Skin & $13(15 \%)$ & 0 & 0 & 0 \\
\hline Hypoglycaemia & $12(14 \%)$ & 0 & $1(1 \%)$ & 0 \\
\hline Hypomagnesaemia & $13(15 \%)$ & 0 & 0 & 0 \\
\hline Anxiety & $12(14 \%)$ & 0 & 0 & 0 \\
\hline Dizziness & $12(14 \%)$ & 0 & 0 & 0 \\
\hline Edema in limbs & $12(14 \%)$ & 0 & 0 & 0 \\
\hline Palpitations & $12(14 \%)$ & 0 & 0 & 0 \\
\hline Bone Pain & $11(13 \%)$ & 0 & 0 & 0 \\
\hline Lung infection & $7(8 \%)$ & $3(4 \%)$ & $1(1 \%)$ & 0 \\
\hline Maculopapular rash & $10(12 \%)$ & $1(1 \%)$ & 0 & 0 \\
\hline Dyspepsia & $10(12 \%)$ & 0 & 0 & 0 \\
\hline Pruritus & $9(11 \%)$ & $1(1 \%)$ & 0 & 0 \\
\hline Creatinine increased & $9(11 \%)$ & 0 & 0 & 0 \\
\hline Hypoalbuminemia & $9(11 \%)$ & 0 & 0 & 0 \\
\hline Musculoskeletal disorder & $9(11 \%)$ & 0 & 0 & 0 \\
\hline Pain & $9(11 \%)$ & 0 & 0 & 0 \\
\hline Abdominal pain & $6(7 \%)$ & $2(2 \%)$ & 0 & 0 \\
\hline GI disorders, other & $7(8 \%)$ & $1(1 \%)$ & 0 & 0 \\
\hline Hypertension & $5(6 \%)$ & $1(1 \%)$ & 0 & 0 \\
\hline Atrial fibrillation & $2(2 \%)$ & $3(4 \%)$ & 0 & 0 \\
\hline Depression & $3(4 \%)$ & $1(1 \%)$ & $1(1 \%)$ & 0 \\
\hline Other infections and infestations & $3(4 \%)$ & $2(2 \%)$ & 0 & 0 \\
\hline Infusion-related reaction & $3(4 \%)$ & $2(2 \%)$ & 0 & 0 \\
\hline Skin infection & $2(2 \%)$ & $2(2 \%)$ & 0 & 0 \\
\hline Syncope & $1(1 \%)$ & $3(3 \%)$ & 0 & 0 \\
\hline Urinary tract infection & $3(4 \%)$ & $1(1 \%)$ & 0 & 0 \\
\hline Dehydration & $2(2 \%)$ & $1(1 \%)$ & 0 & 0 \\
\hline Immune system disorders, other & $2(2 \%)$ & $1(1 \%)$ & 0 & 0 \\
\hline Papulopustular rash & $2(2 \%)$ & $1(1 \%)$ & 0 & 0 \\
\hline Cholecystitis & $1(1 \%)$ & 0 & $1(1 \%)$ & 0 \\
\hline Lipase increased & $1(1 \%)$ & 0 & $1(1 \%)$ & 0 \\
\hline Pneumonitis & $1(1 \%)$ & $1(1 \%)$ & 0 & 0 \\
\hline Sleep apnea & $1(1 \%)$ & $1(1 \%)$ & 0 & 0 \\
\hline Appendicitis & 0 & $1(1 \%)$ & 0 & 0 \\
\hline Arthritis & 0 & $1(1 \%)$ & 0 & 0 \\
\hline Sudden cardiac death & 0 & 0 & 0 & $1(1 \%)$ \\
\hline Gallbladder obstruction & 0 & $1(1 \%)$ & 0 & 0 \\
\hline Pharyngitis & 0 & $1(1 \%)$ & 0 & 0 \\
\hline
\end{tabular}

ALT=alanine aminotransferase. AST=aspartate aminotransferase. GI=gastrointestinal. NOS=not otherwise specified. WBC=white blood cell.

* * In

* Includes pain due to musculoskeletal injuries deemed unrelated to study treatment, muscle cramping, tenderness, or stiffness. 
Table 4:

Non-haematologic grade $\geq 3$ serious adverse events $(\mathrm{N}=85)$

\begin{tabular}{lcc}
\hline & Grade & n (\%) \\
Atrial fibrillation & 3 & $3(4)$ \\
Pneumocystis jiroveci pneumonia & 3 & $1(1)$ \\
Appendicitis & 3 & $1(1)$ \\
Cholecystitis & 4 & $1(1)$ \\
Anaplasmosis infection & 3 & $1(1)$ \\
Pneumatosis intestinalis & 3 & $1(1)$ \\
Lipase elevation & 4 & $1(1)$ \\
Diarrhoea & 3 & $1(1)$ \\
Nausea/vomiting & 3 & $1(1)$ \\
Cellulitis & 3 & $1(1)$ \\
Appendiceal mucocele & 3 & $1(1)$ \\
Respiratory syncytial virus infection & 3 & $1(1)$ \\
\hline
\end{tabular}

